\title{
An outbreak investigation of scrub typhus in Nepal: confirmation of local transmission
}

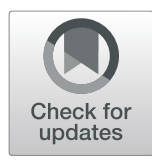

Meghnath Dhimal ${ }^{1 *}{ }^{*}$, Shyam Prakash Dumre ${ }^{2,3 \dagger}$, Guna Nidhi Sharma ${ }^{4,5}$, Pratik Khanal ${ }^{1,6}$, Kamal Ranabhat ${ }^{6,7}$, Lalan Prasad Shah', Bibek Kumar Lal ${ }^{4}$, Runa Jha ${ }^{8}$, Bishnu Prasad Upadhyaya ${ }^{8}$, Bhim Acharya ${ }^{4}$, Sanjaya Kumar Shrestha ${ }^{9}$, Silas A. Davidson ${ }^{10}$, Piyada Charoensinphon ${ }^{10}$ and Khem B. Karki ${ }^{1,6}$

\begin{abstract}
Background: Scrub typhus is a largely ignored tropical disease and a leading cause of undifferentiated febrile illness in the areas of tsutsugamushi triangle caused by Orientia tsutsugamushi. It is frequently diagnosed in South Asian countries, although clear epidemiological information is not available from Nepal. After the 2015 earthquake in Nepal, a sudden upsurge in scrub typhus cases was reported. The objective of this study was to investigate epidemiology of scrub typhus and its causative agents in humans, animals, and chigger mites to understand the ongoing transmission ecology.

Methods: Scrub typhus cases with confirmed diagnosis throughout the country were included in the analysis. Studies were concentrated in the Chitwan district, the site of a major outbreak in 2016. Additional nation-wide data from 2015 to 2017 available from the government database included to analyse the disease distribution by geographical mapping.

Results: From 2015 to 2017, 1239 scrub typhus cases were confirmed with the largest outbreak occurring in 2016 with $831(67.1 \%)$ cases. The case fatality rate was 5.7\% in 2015 which declined to 1.1\% in 2017. A nationwide outbreak of scrub typhus was declared as the cases were detected in 52 out of the 75 districts of Nepal. Seasonal trend was observed with a peak during August and September. In addition to the human cases, the presence of $O$. tsutsugamushi was also confirmed in animals (rodents) and chigger mites (Leptotrombidium imphalum) from the outbreak areas of southern Nepal.

Conclusion: The detection of $O$. tsutsugamushi in humans, animals, and chigger mites from outbreak locations and wide-spread reports of scrub typhus throughout the country consecutively for 3 years confirms the ongoing transmission of $O$. tsutsugamushi with a firmly established ecology in Nepal. The country's health system needs to be strengthened for systematic surveillance, early outbreak detection, and immediate actions including treatment and preventive measures.
\end{abstract}

Keywords: Scrub typhus, Rickettsial infection, Orientia tsutsugamushi, Local transmission, Outbreak, Epidemiology of scrub typhus, Nepal

\footnotetext{
* Correspondence: meghdhimal2@gmail.com

${ }^{\dagger}$ Meghnath Dhimal and Shyam Prakash Dumre contributed equally to this work.

${ }^{1}$ Nepal Health Research Council (NHRC), Ramshah Path, Kathmandu, Nepal Full list of author information is available at the end of the article
}

C C The Author(s). 2021 Open Access This article is licensed under a Creative Commons Attribution 4.0 International License, which permits use, sharing, adaptation, distribution and reproduction in any medium or format, as long as you give appropriate credit to the original author(s) and the source, provide a link to the Creative Commons licence, and indicate if changes were made. The images or other third party material in this article are included in the article's Creative Commons licence, unless indicated otherwise in a credit line to the material. If material is not included in the article's Creative Commons licence and your intended use is not permitted by statutory regulation or exceeds the permitted use, you will need to obtain permission directly from the copyright holder. To view a copy of this licence, visit http://creativecommons.org/licenses/by/4.0/ The Creative Commons Public Domain Dedication waiver (http://creativecommons.org/publicdomain/zero/1.0/) applies to the data made available in this article, unless otherwise stated in a credit line to the data. 


\section{Background}

Scrub typhus is a vector-borne acute febrile illness caused by Orientia tsutsugamushi and transmitted to humans and rodents by infected chigger mites (larval stage of Trombiculidae mites) [1, 2]. Historically, scrub typhus had been endemic in Asia, Australia and islands in the Indian and Pacific Oceans, known as the "tsutsugamushi triangle" [1]. However, there have been recent reports of scrub typhus from Africa, the Middle East, and South America suggesting the disease is no longer restricted to this triangle [2] but no autochthonous cases have been reported from north America and Europe. Scrub typhus is frequently reported from many Asian countries and is endemic in Nepal's neighboring countries including India (Sub-Himalayan belt) and Bhutan, where it is considered an emerging infectious disease [3-6]. In Bhutan, one in six undifferentiated febrile patients had rickettsial infections, with scrub typhus being the most common [6]. However, the disease situation in Nepal remained unexplored until 2015, likely due to the high burden of other febrile illnesses with indistinguishable clinical signs and limited availability of diagnostics.

There have been a few previous attempts to investigate the incidence of scrub typhus in Nepal. As early as 1981, a study showed the high possibility of scrub typhus in Nepal when it demonstrated elevated antibody titers in $10 \%$ of healthy adults [7]. Unfortunately, additional surveillance studies in the country were not conducted for next 23 years until 2004. A serological investigation of scrub typhus at Patan Hospital, Kathmandu found a small number of febrile patients (28/876) positive for scrub typhus antibodies [8]. The investigation was performed using a multi-test assay without further confirmation for scrub typhus by immuno-fluorescent assay (IFA), gold standard for scrub typhus diagnosis. It was inconclusive whether scrub typhus or another rickettsial illness, such as murine typhus, was present. Another report in 2007 also indicated the presence of scrub typhus in Nepal [9]. However, no outbreak investigations of scrub typhus (with fatality information) were reported in Nepal before 2015 and no systematic investigations by the government had been conducted. As a consequence, scrub typhus cases had not been reported to the Epidemiology and Disease Control Division (EDCD) of the Ministry of Health and Population before 2014 [10].

In April 2015, Nepal experienced a mega-earthquake claiming thousands of lives, massive destruction, and huge economic losses followed by an upsurge in febrile illnesses [11, 12]. Three months after this devastating earthquake in Nepal (August 2015), a tertiary care teaching hospital in Nepal alerted EDCD that children with fever and severe respiratory features were not responding to the usual course of antibiotic treatment, leading to high mortality rates $(8 \%)[10,11]$. The clinicians had used cefexime or ceftraiaxone and imipenem in intensive care. After the initially suspected aetiologies (hantavirus and other viral diseases) were ruled out, the samples were screened with M- Enzyme Linked Immunosorbent Assay (ELISA) for scrub typhus and found positive. This was the first and most significant fatal scrub typhus outbreak in the country [11]. Since then, scrub typhus has been increasingly reported in Nepal but no clear epidemiological picture is available.

In this study, a systematic investigation was carried out in Nepal to investigate the ongoing transmission ecology of Scrub typhus which included patient, vector, and animal studies employing both serological and molecular tools.

\section{Methods}

\section{Study design and settings}

This was a descriptive cross-sectional study conducted in Nepal and included scrub typhus cases of any age and sex reported in 2016 from 52 districts out of 75 districts the country. Nepal has three distinct ecological regions from north to south, namely: mountain, hill and terai (plain area). Muntain region is comprised of high mountains in the north including the top of the world while hilly regions consists of mid-hills between mountains and terai (low-land). Terai region is the southern lowland ecological trails spanning from east to west.

Serologically confirmed cases of scrub typhus from various government authorized laboratories, including National Public Health Laboratory (NPHL), were reported to EDCD and compiled in the database of EDCD. As part of the outbreak investigation, chigger and animal studies were also carried out in the major outbreak areas in Chitwan district as detailed below. Additionally, the aggregated data from 2015 and 2017 were used for mapping. An overall flow diagram of the study is detailed in Fig. 1.

\section{Case definitions}

The patients were classified based on the EDCD Guideline on Prevention and Control of scrub typhus adopted from the World Health Organization (WHO) [10]. Briefly, 1) a case with acute undifferentiated febrile illness (AUFI) of $\geq 5$ days with or without eschar (fever of $<5$ days for a case with eschar) was considered a "suspected/clinical case"; 2) a suspected/clinical case with an IgM titer > 1:32 and/or a four-fold increase of titers between acute and convalescent sera was considered to be a "probable case"; and 3) a "confirmed case of scrub typhus" was declared when the O. tsutsugamushi DNA was detected in eschar or whole blood samples by polymerase chain reaction (PCR), or four-fold rise in antibody titers on acute and convalescent sera by IFA, 


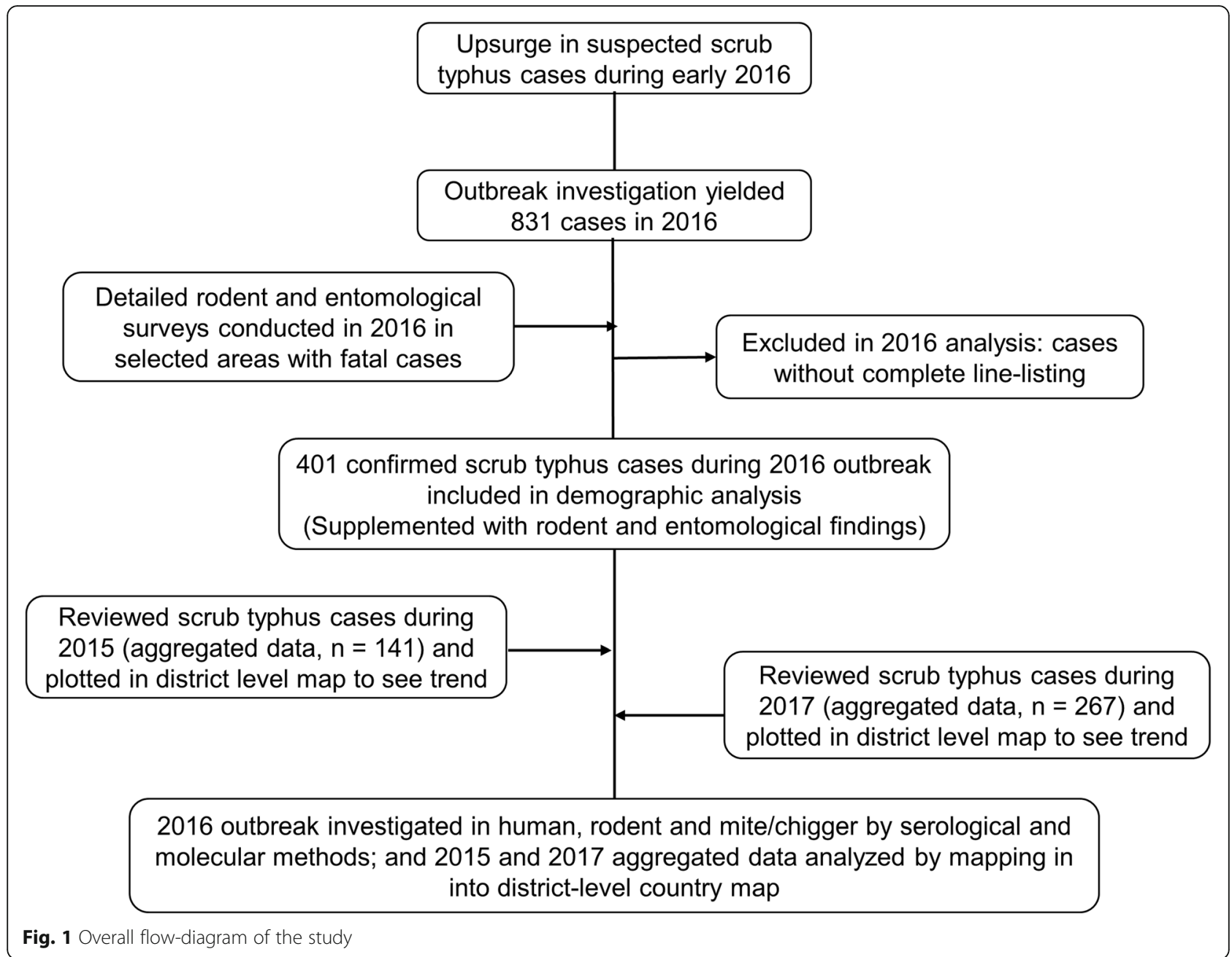

which is the gold standard assay, or Indirect ImmunoPeroxidase (IIP) assay.

Human sample collection and laboratory investigation for O. tsutsugamushi infection

The Scrub Typhus Detect ${ }^{\mathrm{TM}}$ IgM Rapid Test kits were used for the detection of IgM antibodies to members of O. tsutsugamushi species in human blood of all cases visiting periphery level health care centers. For outbreak investigation purpose, blood samples were collected from the suspected scrub typhus cases presented to the NPHL, Kathmandu, and from Chitwan Medical College (CMC) Hospital and Bharatpur Hospital, Chitwan. Samples collected from sentinel sites including $\mathrm{CMC}$ and Bharapur Hospital were transferred to NPHL, maintaining the cold chain. Samples were tested for scrub typhus using an O. tsutsugamushi specific IgM-ELISA (Scrub Typhus Detect ${ }^{\mathrm{TM}}$ Kit, InBios International, WA, USA) and interpreted as per the manufacturer's manual. The remaining aliquots of the sample were cryo-preserved at $-80^{\circ} \mathrm{C}$. Representative scrub typhus IgM positive and negative serum samples (confirmed by IgM-ELISA) were further confirmed by IFA using a panel of O. tsutsugamushi specific antigens covering major O. tsutsugamushi strains identified in endemic areas at the Armed Forces Research Institute of Medical Sciences (AFRIMS), Bangkok, Thailand and Walter Reed/ AFRIMS Research Unit Nepal (WARUN) as described previously [13, 14]. Briefly, pooled antigens from whole cell-cultured prototype strains including Karp, Kato, and Gillian were used to detect $O$. tsutsugamushi specific antibodies in patient serum samples. An initial screen was performed using dilution of 1:50. Any positive sample was then 2-fold serially diluted to a final concentration of 1:12,800 and tested for antibody titer. FITC-conjugated rabbit antihuman IgG (Rabbit anti-human IgG-FITC secondary Antibody) was used to determine O. tsutsugamushi specific antibodies in humans. Serum titers less than 1:50 were interpreted as negative for IgM and IgG, whereas serum titers 1:50 and less than 1:400 indicated past or recent infection, and serum titer equal to or higher than 1:400 indicated active infection. This cutoff is a reference 
value currently used by several research institutes including National Institute of Health (http://nih.dmsc. moph.go.th/login/showimgdetil.php?id=514) and Ministry of Science and Technology, Thailand. The epidemiological characteristics of confirmed patients such as age, sex, ethnicity, month of diagnosis, ecological region, administrative region and clinical outcome was recorded. Out of the total 831 cases reported in 2016, complete demographic data requested was only available for 401 cases.

\section{Animal and entomological investigation of scrub typhus outbreak \\ Site selection}

Chitwan district was selected for animal and entomological surveillance based on the severity and prevalence of the disease in and around this district. Chitwan district belongs to terai region which is the low-land plain region (elevation as low as $70 \mathrm{~m}$ above mean sea level) situated at the southern belt of Nepal (south of the outer foothills of Himalayas) and bordering to India. Terai has warmer and humid climate and is endemic for many vector-borne tropical disease. Fatalities due to scrub typhus mainly occurred in the Mangalpur Village Development Committee
(VDC), which is a sub-district level administrative area of Chitwan district. Therefore, three VDCs (Mangalpur, Sharadanagar and Shukranagar), which are located to the South-West of Bharatpur (the district headquarters), were selected for the surveillance study (Fig. 2).

\section{Animal capture}

Rodent and shrew trapping was performed in and around the houses of the index cases and their neighbors. They were trapped using Sherman traps tagged with a unique number for identification as described previously [14]. All the traps were equipped with different baits (ripe banana, tomato, or piece of chicken) and placed at various sites $(n=104)$ inside and outside houses, cattle sheds, near granaries and the nearby fields (e.g. kitchen gardens) following the trap lines. Traps were set in the early evening and collected the following morning. A total of 104 traps were placed and 12 animals (nine rodents and three shews) were captured.

\section{Animal blood collection and species identification}

The animals, still in the trap, was placed inside a gas chamber and anaesthetized using carbon dioxide inhalation method $\left(2-3 \mathrm{~L} / \mathrm{h}\right.$, maintaining $30 \%$ initial $\left.\mathrm{CO}_{2}\right)$.

\section{NEPAL - Chitwan District}

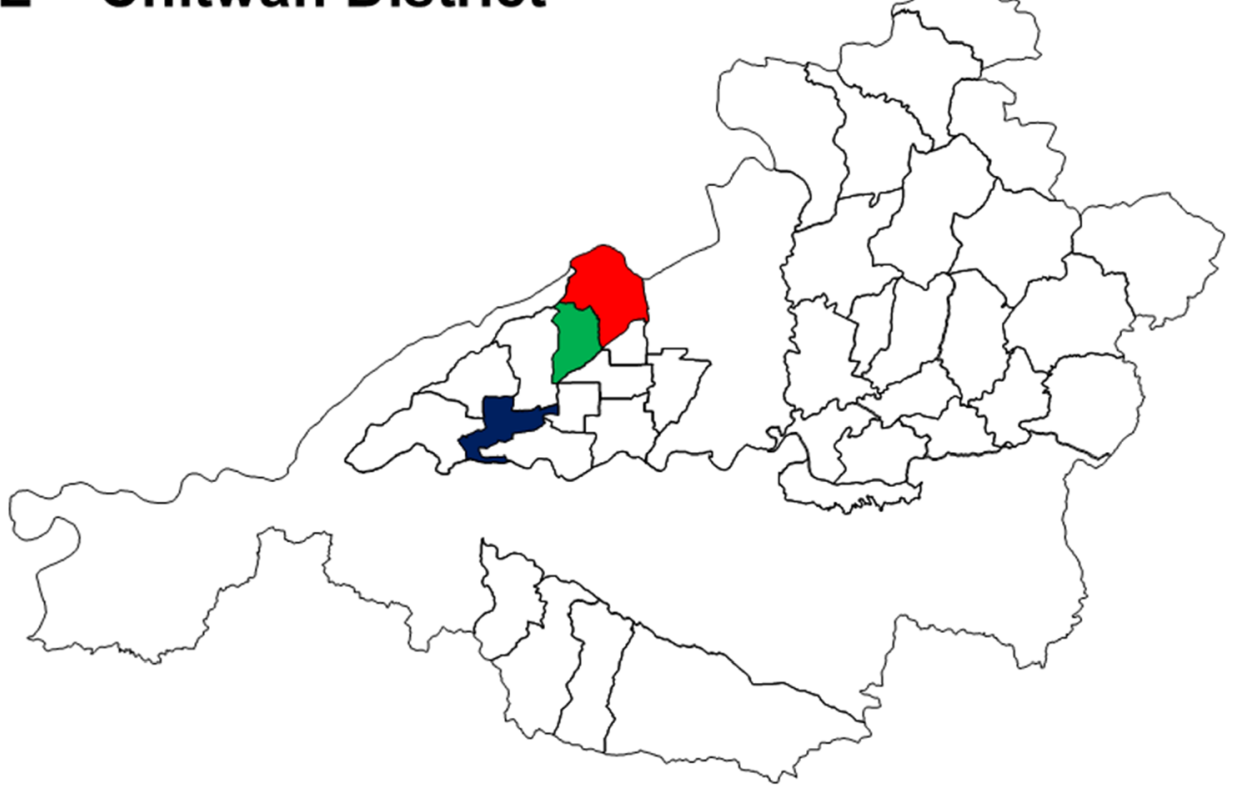

Fig. 2 Map of Chitwan district showing the animal and entomological study sites. Investigation was carried out in three VDCs (Mangalpur, Sharadanagar and Shukranagar) (dark filled areas) which are located to the South-West of the district headquarters of Chitwan (the outbreak epicenter district). VDC, village development committee (Source: EDCD data using Arc GIS software) 
Following the euthanasia, the animal was taken out from the trap, weighed, and essential characteristics were recorded for species identification. Blood was collected by direct cardiac puncture using $3 \mathrm{ml}$ disposable syringes. Serum samples pipetted into cryo-tubes were kept in a cold box with pre-frozen ice packs at $-80^{\circ} \mathrm{C}$ and immediately shipped to the laboratory of WARUN at Bharatpur Hospital and then to WARUN in Kathmandu in cold box where the samples were stored at $-80^{\circ} \mathrm{C}$ until analyzed. The samples were analyzed at WARUN for further related investigations. Rodent and shrew species were identified by their physical appearance, morphology, length of head and body, tail as well as ear/pinna, color patterns of dorsal and ventral fur, number of mammary glands.

\section{Mite collection, identification and processing}

Mites and other ectoparasites were collected by combing the anaesthetized animal's outer surfaces (ventral and dorsal surfaces, arm-pits, groins, and areas near pinnas etc.) onto a clean, white sheet of paper to help identify the ectoparasites. The ear pinna of animals was examined under a dissecting microscope for chiggers and individual or clusters of chiggers were removed together with a thin layer of ear skin using fine forceps. Chiggers were transferred into labelled vials containing 70\% ethanol and stored in an ice-cold box for preservation. Representative chiggers from each animal host were randomly sampled for morphological identification. Permanent slides of chigger samples were prepared and mounted at WARUN laboratory in Kathmandu, Nepal following the method described previously [15]. The animal sera were tested for the presence of IgG antibody against $O$. tsutsugamushi by IFA. Moreover, the O. tsutsugamushi specific quantitative PCR (qPCR) as performed in animal tissue samples and chigger samples as described previously [14].

\section{Data analysis}

Data were entered in Microsoft Excel and analyzed in IBM SPSS version 22.0. Descriptive analysis was done by calculating frequency and percentages for categorical data. Data were analyzed by chi-square tests for assessing significant difference in proportions across groups. To understand the nationwide situation of scrub typhus, cases were plotted onto the district-level country maps.

\section{Ethics}

This study was approved by the Ethical Review Board of Nepal Health Research Council (NHRC), Government of Nepal (Reg. No 305/2016) which approves health research involving human and animal subjects in Nepal. This study was conducted following National Ethical Guidelines for Health Research in Nepal and Standard Operating Procesuure 2011 and Ethical Guidelines for the Use and Care of Animals in Health Research in Nepal 2005. All patient records were anonymized before analysis. All procedures involving animals were carried out as per the Ethical Guidelines for the Care and Use of Animals in Health Research in Nepal- 2005 and followed the regulation of local and national laws regarding the use of animals for research purpose [16].

\section{Results}

\section{Socio-demographic findings}

In 2016, a total of 831 cases with 14 deaths (case fatality rate $(\mathrm{CFR})=1.7 \%$ ) were reported from 47 districts throughout the country (Fig. 3). Although 831 cases were reported in the country during April to December 2016 in Nepal, a complete line listing was available for only 401 cases $($ male $=163$; female $=238)$ from EDCD. The mean age (years) \pm standard deviation of the cases was $28.9 \pm$ 18.2 years and median was 25.0 (IQR 14.0-42.0) years. More than half of the cases (57.1\%) were below 30 years of age; of these $14.5 \%$ were children below 10 years of age (Table 1). The majority of the cases belonged to Janajati/ Aadhibasi (44.4\%) and Brahmin/Chhetri (44.1\%) ethnic groups with no gender-based differences.

Overall, young adults and children were the majority affected by scrub typhus in Nepal, and this proportion significantly differed by gender $(p<0.001)$ (Table 1$)$. Among male scrub typhus patients, the age skewed equally towards children below 10 years $(22.7 \%)$ and 10 19 years $(22.7 \%)$ while among females, more cases were in the age group $20-29$ years (26.9\%). There was no difference in the median age of scrub typhus patients by gender $(p=0.237)$ (Table 1$)$.

\section{Geographical and temporal distribution of scrub typhus in Nepal, 2016}

The vast majority of scrub typhus cases were from Tarai (81.5\%; $p=0.03)$, the low-land ecology of southern Nepal bordering northern India. The regional analysis identified the central region (45.6\%) as the most affected area in the country (Table 1, Fig. 3). The most affected district was Chitwan, contributing $34.4 \%$ of the total cases $(n=138)$, followed by Kailali $(n=63)$, Nawalparasi $(n=$ $55)$, Kanchanpur $(n=26)$, Gorkha $(n=15)$ and Tanahun $(n=10)$ (Fig. 3, Suppl Table 1). The 2016 outbreak of scrub typhus demonstrated a seasonal trend in Nepal that peaked in the months of August $(n=164 ; 40.9 \%)$ and September $(n=105 ; 26.2 \%)(p=0.01)$ (Table 1$)$.

\section{Diagnosis, outcome and trend of scrub typhus cases in Nepal, 2015-2017}

In 2016, cases were reported or confirmed at five health facilities (Suppl Table 1) mostly from the outbreak epicenter, Chitwan district (61.3\%). Representative serum samples $(n=61)$ tested by IFA further confirmed the 


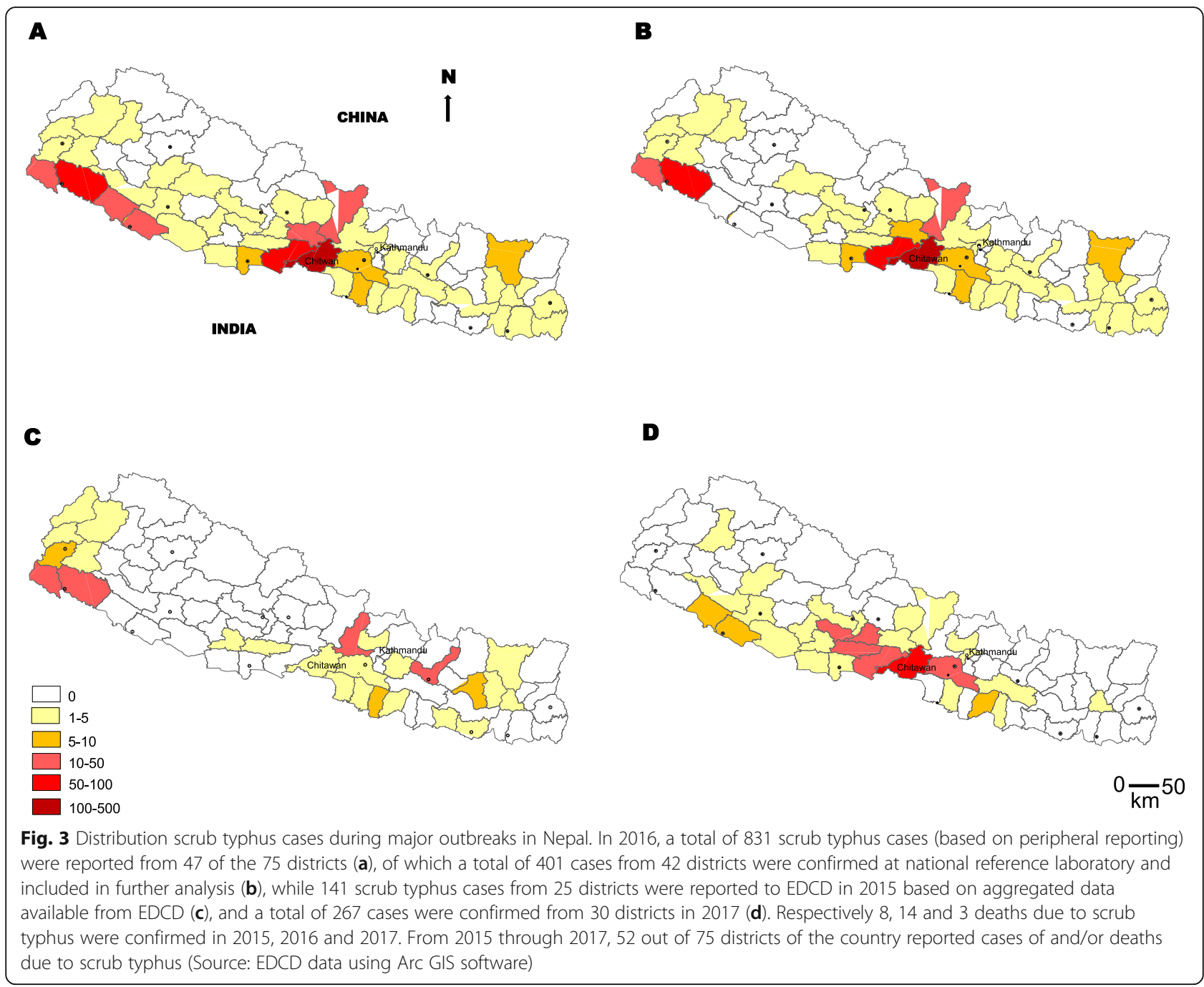

presence of $O$. tsutsugamushi infection in Nepal (Table 2). From the 33 samples which tested positive by ELISA, 28 also tested positive by IFA. Similarly, one of the 28 ELISA negative sample was positive by IFA. Regarding the prognosis of the disease, 12 cases (3\%) $(6$ males and 6 females) died while the rest recovered after treatment. After analyzing the cases reported to EDCD during 2015 to $2017(n=1239)$, it was determined that the disease was firmly established in Nepal, and a large outbreak occurred in $2016(n=831)$ involving 47 districts. An additional 267 cases were reported in 2017 and there were 141 reported cases in 2015 (Table 3). Twenty-five people died as a result of scrub typhus during 2015-2017 with an overall CFR of 2.0, which decreased from 5.7 in 2015 to 1.1 in 2017 (Table 3).

\section{Animal and entomological investigation identified hosts} of O. tsutsugamushi in the outbreak areas of Nepal Of the total 104 traps used, 12 animals (9 the Rattus rattus species complex and 3 shrews named Suncus murinus) were successfully trapped. (Suppl Table 2). Out of 12 animals, three (25\%) had chigger mite infestation. The chigger index was 0.92. Details of animals and chiggers have been presented in Suppl Tables 2 and 3.

\section{Confirmation of $O$. tsutsugamushi infection in animals and chiggers collected in the outbreak areas of Nepal by IFA/ molecular techniques}

Three animal serum samples out of nine were confirmed scrub typhus positive by IFA (IgG titer $>50$ ) (Suppl Table 3). However, all the 24 animal tissue samples were O. tsutsugamushi negative by PCR. Similarly, one of the three chigger (Leptotrombidium imphalum) samples was also confirmed O. tsutsugamushi positive by PCR.

\section{Discussion}

Our study demonstrates that scrub typhus is an emerging public health problem in Nepal with several outbreaks since 2015. Scrub typhus is an under studied 
Table 1 Epidemiological characteristics of scrub typhus cases in Nepal, 2016*

\begin{tabular}{|c|c|c|c|c|}
\hline Variables & $\begin{array}{l}\text { Male } n(\%) \\
n=163\end{array}$ & $\begin{array}{l}\text { Female } n(\%) ; \\
n=238\end{array}$ & $\begin{array}{l}\text { Total } n(\%) ; \\
n=401\end{array}$ & $p$-value \\
\hline Age in years, median (IQR) & $24.0(11.0-50.0)$ & $25.0(17.0-39.0)$ & $25.0(14.0-42.0)$ & 0.237 \\
\hline \multicolumn{5}{|l|}{ Age group } \\
\hline Below 10 & $37(22.7)$ & $21(8.8)$ & $58(14.5)$ & \multirow[t]{7}{*}{$<0.001$} \\
\hline $10-19$ & $37(22.7)$ & $51(21.4)$ & $88(21.9)$ & \\
\hline $20-29$ & $19(11.7)$ & $64(26.9)$ & $83(20.7)$ & \\
\hline $30-39$ & $16(9.8)$ & $44(18.5)$ & $60(15.0)$ & \\
\hline $40-49$ & $11(6.7)$ & $21(8.8)$ & $32(8.0)$ & \\
\hline $50-59$ & $28(17.2)$ & $25(10.5)$ & $53(13.2)$ & \\
\hline 60 and above & $15(9.2)$ & $12(5.0)$ & $27(6.7)$ & \\
\hline \multicolumn{5}{|l|}{ Children vs adults } \\
\hline 15 years and below & 63 & 54 & 117 & \multirow[t]{2}{*}{0.001} \\
\hline Above 15 years & 100 & 184 & 284 & \\
\hline \multicolumn{5}{|l|}{ Ethnicity } \\
\hline Brahmin/Chhetri & $72(44.2)$ & $105(44.1)$ & $177(44.1)$ & \multirow[t]{5}{*}{0.87} \\
\hline Janajati/Aadhibasi & $74(45.4)$ & $104(43.7)$ & $178(44.4)$ & \\
\hline Madheshi & $5(3.1)$ & $5(2.1)$ & $10(2.5)$ & \\
\hline Dalit & $9(5.5)$ & $18(7.6)$ & $27(6.7)$ & \\
\hline Others & $3(1.8)$ & $6(2.5)$ & $9(2.2)$ & \\
\hline \multicolumn{5}{|l|}{ Month (seasonality) } \\
\hline June & $12(7.4)$ & $5(2.1)$ & $17(4.2)$ & \multirow[t]{5}{*}{0.01} \\
\hline July & $27(16.6)$ & $29(12.2)$ & $56(14.0)$ & \\
\hline August & $71(43.6)$ & $93(39.2)$ & $164(41.0)$ & \\
\hline September & $36(22.1)$ & $69(29.1)$ & $105(26.2)$ & \\
\hline October & $17(10.4)$ & $41(17.3)$ & $58(14.5)$ & \\
\hline \multicolumn{5}{|l|}{ Ecological region } \\
\hline Mountain & $8(4.9)$ & $2(0.8)$ & $10(2.5)$ & \multirow[t]{3}{*}{0.03} \\
\hline Hill & $28(17.2)$ & $37(15.5)$ & $65(16.2)$ & \\
\hline Terai & $127(77.9)$ & 199 (83.6) & $326(81.3)$ & \\
\hline \multicolumn{5}{|l|}{ Administrative region } \\
\hline Eastern & $12(7.4)$ & $6(2.5)$ & $18(4.5)$ & \multirow[t]{4}{*}{0.08} \\
\hline Central & $75(46.0)$ & $107(45.0)$ & $182(45.4)$ & \\
\hline Western & 39 (23.9) & $56(23.5)$ & $95(23.7)$ & \\
\hline Mid-Far West & $37(22.7)$ & $69(29.0)$ & $106(26.4)$ & \\
\hline \multicolumn{5}{|l|}{ Clinical outcome } \\
\hline Cured & $157(96.3)$ & $232(97.5)$ & $389(97.0)$ & \multirow[t]{2}{*}{0.50} \\
\hline Death (CFR) & $6(3.7)$ & $6(2.5)$ & $12(3.0)$ & \\
\hline
\end{tabular}

*Scrub typhus patients with complete line-listing information $(n=401)$ were included in this analysis IQR Inter-quartile range; CFR Case fatality rate

neglected tropical disease and a leading cause of undifferentiated treatable fever in Asia [1, 17]. This study uncovered the firmly established nature of scrub typhus outbreaks in Nepal through evidence of the causative agent $O$. tsutsugamushi in human (patients), animals and vector/reservoir hosts (chigger mites) during the recent fatal outbreaks.

Despite the frequent reports of outbreaks from neighbouring countries, particularly India [3-6, 18-21], scrub typhus was undetected in Nepal for decades after its first 
Table 2 Laboratory findings of selected human samples underwent IFA confirmation

\begin{tabular}{lllll}
\hline Sample collection sites & Total samples & Positive by RDT (\%) & Positive by IgM ELISA (\%) & Positive by IFA (\%) \\
\hline NPHL & 50 & NT & $30(60.0)$ & $26(52.0)$ \\
Bharatpur hospital & 11 & $3(27.3)$ & NT & $3(27.3)$
\end{tabular}

A fraction of samples $(n=61)$ from suspected scrub typhus cases were randomly selected and sent to reference laboratory (Armed Forces Research Institute of Medical Sciences, Bangkok, Thailand) for IFA confirmation. RDT, rapid diagnostic test; ELISA Enzyme linked immune-sorbent assay; IFA, Indirect fluorescence antibody assay; NPHL National Public Health Laboratory; NT Not tested

indication in 1981 [7]. This has resulted in a significant lack of understanding when it comes to epidemiological features, treatment response and severity, and its ecological niche. This type of baseline information is required for a country to formulate appropriate guidelines and pave strategies for scrub typhus control. Apart from a handful of reports discussing the human cases [7-10, 22, 23], no study has explored whether the human-hostvector/reservoir-pathogen cycle is maintained in Nepal. This ecological chain is essential to understand a sudden outbreak of any magnitude [24]. The present study has contributed by providing solid evidence of the ongoing circulation of the scrub typhus pathogen O. tsutsugamushi among human, animals, and chigger mites in areas affected by recent large outbreaks.

The scrub typhus outbreaks that occurred in Nepal during 2015 to 2017 may be linked to the devastating earthquake of 2015. The outbreaks could have been triggered as a result of intimate contact between humans and mite infested rats that might have come out of their usual underground habitat with the demolition of many houses [25], and this would provide people increased exposure to $O$. tsutsugamushi infected mites which are both the vectors and the reservoirs. However, there is no evidence to support this hypothesis. Close proximity while living in temporary shelters [12] due to overcrowding and unsanitary conditions could have contributed to increased contact between vectors, pathogens and humans [25]. A weakened health system due to the massive earthquake compromised the availability of diagnostic and treatment facilities, which further affected the control program resulting in the large outbreak in 2016.

A large scrub typhus outbreak during 2015 to 2017 initiated in 2015 with 141 cases and nine deaths, which was an unforeseen eruption of this disease after the years of silence in the country. The apparent disappearance of the disease in a territory for a long period before a sudden re-emergence in an epidemic form is symptomatic of scrub typhus. For example, scrub typhus re-emerged in the Maldives as a fatal epidemic in 2002-2003 after 58 years of its disappearance [26, 27], and it also resembles the scenario in India where the re-emergence was observed during 1990s after World War II [4]. There was no strong evidence of persistent scrub typhus in Nepal after the initial indication in 1981, although we cannot totally exclude this possibility considering the lack of diagnostic facilities, endemicity, and inadequate clinical suspicion/precision in Nepal. In peripheral settings of Nepal, widal test is exclusively used for enteric fever due to the lack of blood cultures facilities. This may lead to misdiagnosis of enteric fever [12], one of the most common AUFI [28], when the actual etiology might be other febrile diseases like scrub typhus, leptospirosis, dengue, etc. Such misdiagnosis [12] and the minimal clinical interest (due to effective treatment) could be other factors for no visible infections [26]. Nevertheless, we cannot overlook the serologically positive cases reported in 2004 and 2007 in the Kathmandu valley $[8,9]$ while considering such absence of the overt disease in this location.

Among those infected with scrub typhus, the majority were females aged below 40 years, with a significant proportion of children. Younger and reproductive females in rural areas are mostly involved in outdoor or agriculture activities in Nepal,and this could be one reason for increased scrub typhus infection. India, South Korea and China have also reported higher incidence among females $[18,29,30]$. Similarly, we observed a clear seasonality with the majority of cases being detected in August and September, which is quite similar to what was reported from the Indian states $[18,21]$. The overall pattern of scrub typhus in Nepal resembles that in neighbouring countries including India, indicating

Table 3 Trend of scrub typhus cases and deaths in Nepal, 2015-2017

\begin{tabular}{lllll}
\hline Year & No. of districts & $\begin{array}{l}\text { No. of cases } \\
\text { (\%) }\end{array}$ & No. of deaths (\%) & Case fatality rate, \% \\
\hline 2015 & 25 & $141(11.4)$ & $8(32.0)$ & 5.7 \\
2016 & 47 & $831(67.1)$ & $14(56.0)$ & 1.7 \\
2017 & 30 & $267(21.5)$ & $3(12.0)$ & 1.1 \\
Cumulative, 2015-2017 & 52 & $1239(100)$ & $25(100)$ & 2.0 \\
\hline
\end{tabular}


potential cross-border transmission due to massive trade and transport activities (woods, trucks) and lack of physical barrier probably provide ample opportunity for chiggers' migration across the borders .

In Nepal, scrub typhus cases were reported nationwide (52 of 75 districts) in just 3 years, and the disease may expand very rapidly as seen in other parts of the world [31, 32]. In this study, the majority of cases were reported from lowland terai districts where other febrile illnesses including Japanese encephalitis (JE) [33, 34], leptospirosis [35], and dengue [33, 34, $36,37]$ have been frequently reported. Even before the initiation of JE vaccination in Nepal, only onethird of the acute encephalitis syndrome (AES) cases were due to JE, which declined with immunization. However the AES remains persistent, clearly indicating other aetiologies of AES in the country. From the same areas of Nepal, cases of leptospirosis and dengue were identified among AES population [34, 35]. Looking at the severity and high fatality (up to 6\%) of scrub typhus during 2015 to 2017, it is logical to consider that a fraction of AES cases could be due to scrub typhus and vice-versa in Nepal. This is further supported by the contemporaneous outbreaks occurring in some states of India (along the Nepal border) where scrub typhus was identified as one of the significant causes of AES [20, 24]. Interestingly, six out of eight fatal cases reported in that Indian state had evidence of O. tsutsugamushi infection [20]. In addition to AES cases, rodents and mites were also positive for $O$. tsutsugamushi in those AES-reported areas [24]. This is similar to what we found in Nepal, suggesting the need to include scrub typhus in the differential diagnosis in AES and other acute undifferentiated febrile illness (AUFI) which are common in Nepal [38].

Despite the high fatality rate (6\%) observed during the first wave of scrub typhus in Nepal in 2015, CFR successfully declined to approximately $1 \%$ in 2017 , which is quite encouraging for the disease control program. This rate is within the wide range of reported fatality (median, 6 and 1.4\% for untreated and treated cases, respectively) [1]. The government initiatives that may have reduced the CFR $[10,11]$ include distribution of guidelines, public awareness, and training and orientation of health workers for prompt treatment of scrub typhus with available drugs (doxycycline, azithromycin, and chloramphenicol or their appropriate combinations). Infection with resistant or reduced drug-susceptible $O$. tsutsugamushi strains often yield very high mortality (up to $24 \%)$, miscarriage and poor neonatal outcomes [1]; it may be speculated that these strains are yet to emerge in Nepal. Since there was no definitive evidence for this, careful monitoring for drug resistance (including potential resistance genes) should be in place. Poor response with doxycycline in some countries suggested the potential emergence of resistant strains [26]. Early administration of doxycycline/azithromycin reduced the progression to AES in India [3], and so treatment delay should be avoided.

This study has some limitations. The detailed individual level data were not available for cases of 2015 and 2017, and even all cases of 2016 was not available for analysis which suggest the need of sustainable integrated surveillance system. The IFA and PCR confirmation tests were limited to representative samples, and further genetic characterization of the pathogenic strain of O. tsutsugamushi that circulated in Nepal was not performed. Although a total of 104 animal traps were set in the suspected areas, a relatively small number of animals were captured as well as a low number of chigger mites (L. imphalum). We cannot discount the possibility of other animal species associated with scrub typhus in Nepal. Although we did not cover other rickettsial diseases, cases of spotted fever group, typhus group and Q Fever rickettsia in scrub typhus endemic areas of neighbouring countries like India [19] and Bhutan [6] warrant further investigation of these aetiologies in Nepal. Recent indications of $\mathrm{Q}$ fever in some acute undifferentiated febrile cases in Nepal also underscores this [22]. Apart from humans, very high rickettsial seropositivity was reported among domestic animals in Bhutan suggesting that a One Health approach could be useful in understaning the prevalence of $O$. tsutsugamushi through serological sentinel surveys that may aid in rickettsial diseases control programs [39]. Therefore, the need for domestic animal studies is also key in the context of Nepal.

\section{Conclusion}

The outbreak investigation in humans, animals and chigger mites with positive serological and molecular evidence, and an analysis of additional aggregated data confirm a firmly established scrub typhus infection ecology in Nepal. This is evidence of ongoing transmission of O. tsutsugamushi in Nepal. Scrub typhus cases were reported throughout the country for 3 years after the 2015 earthquake and this underlines the risk for outbreaks of epidemic potential. Establishment of a sufficient reference laboratory with IFA and molecular facility is urgently required in the country for confirmation, genetic characterization for detection of numerous strains in circulation and evolutionary analysis. Most importantly, the health system needs to be strengthened for systematic surveillance, early outbreak detection and immediate responses including treatment and preventive measures in the country. 


\section{Supplementary Information}

The online version contains supplementary material available at https://doi. org/10.1186/s12879-021-05866-6.

Additional file 1: Supplementary information on extended methodological details on animal and entomological investigations. Supplementary information on findings of animal and entomological investigations. Supplementary Table 1. Distribution of Scrub typhus cases in in Nepal, 2015-2017. Supplementary Table 2. Characteristic features of animals captured during scrub typhus outbreak in Nepal. Supplementary Table 3. Serological (IFA) and molecular findings of animals and chigger samples during scrub typhus outbreak in Nepal.

\section{Abbreviations}

AES: Acute encephalitis syndrome; AFRIMS: Armed forces research institute of medical sciences; CFR: Case fatality rate; CMC: Chitwan Medical College; EDCD: Epidemiology and Disease Control Division; JE: Japanese encephalitis; IFA: Immuno-fluorescent assay; IgM-ELISA: Immunoglobin M- enzyme linked immunosorbent assay; IIP: Indirect immuno-peroxidase; NHRC: Nepal Health Research Council ; NPHL: National Public Health Laboratory; PCR: Polymerase Chain Reaction; VDC: Village Development Committee; WARUN: Walter Reed Afrims Research Unit Nepal; WHO: World Health Organization

\section{Acknowledgements}

Authors thank Dr. Baburam Marasini, then Director of EDCD and Dr. Krishna Kumar Aryal, former Research Officer of NHRC for their contribution in research design. Similarly, we applaud the efforts of Mr. Resham Lamichhane, Mr. Uttam Raj Pyakurel and Mr. Bijay Rimal from EDCD, Mr. Shishir Kumar Pant from Vector Borne Disease Research and Training Centre (VBDRTC), Hetauda, Mr. Pramod Kumar Mehta from Shukraraj Tropical \& Infectious Disease Hospital-Teku, Mr. Laxman Maharjan, and Mr. Buddha Ratna Maharajan from NPHL and Mr. Bijay Kumar Jha, Mr. Ram K.C. from District Public Health Office, Chitawan, Dr. Dayaram Lamsal, Dr. Santosh Pathak \& Mr. Sanjay Yadav from CMC Hospital, Bharatpur for their co-operation to conduct this study.

We are very grateful Dr. Wuttikon Rodk Vamtook, Mr. Surachai Leepita Krat and Ms. Maneerat Somsri of AFRIMS, Thailand as WHO Collaborating Centre for Diagnostic Reference, Training and Investigation of Emerging Infectious Diseases for SEARO and Ms. 'Jasmine Shrestha, Mr. Pashupati Khanal, Mr. Ashish Shrestha, Mr. Bishnu Bahadur Rayamajhi, Ms. Samita Bajracharya \& Ms. Bina Sakha of WARUN for their technical support in confirmatory diagnosis of human samples and rodent/mite investigations. We woud also thank Ms. Anna Durcane Bagale of London School of Hygiene and Tropical Medicine for reviewing the manuscript.

\section{Authors' contributions}

$M D, K R, G N S$ and KBK initially conceptualized the paper, developed the study protocol and finalized the manuscript. SPD, PK, KR and MD performed data analysis and wrote the first draft of the manuscript. LPS, BPU and SKS conducted the field study while BA, BKL and RJ critically reviewed the draft manuscript. SAD and PC contributed to the study design and interpretation of data. All authors read and approved the final manuscript.

\section{Funding}

This research study was supported by Nepal Health Research Council (NHRC) and Epidemiology and Disease Control Division (EDCD), Department of Health Services. Both funding organizations received funding from the Government of Nepal and the Government of Nepal itself did not have any role in the research process including study design, data management and manuscript writing.

\section{Availability of data and materials}

The datasets used and/or analysed during the current study are available from the corresponding author on reasonable request.

\section{Ethics approval and consent to participate}

This study was approved by the Ethical Review Board of Nepal Health Research Council, Governemnt of Nepal (Reg. No 305/2016). The suspected patients were diagnosed for Scrub typhus as a part of the routine clinical care. The Epidemiology and Disease Control Division (EDCD) compiled the individual's information in their database which was then provided to the research team after administrative approval. Hence, the research team did not have direct contact with the patients and hence, consent was not required with individuals. However, all patient records were anonymized before analysis by the research team. All procedures involving animals were carried out as per the Ethical Guidelines for the Care and Use of Animals in Health Research in Nepal and followed the regulation of local and national laws regarding the use of animals for research purpose. Beside ethical approval from national regulatory body, additional approval for collection of animal sample for this study was not needed.

\section{Consent for publication}

Not Applicable.

\section{Competing interests}

We declare no competiting interests.

\section{Author details}

${ }^{1}$ Nepal Health Research Council (NHRC), Ramshah Path, Kathmandu, Nepal. ${ }^{2}$ Central Department of Microbiology, Tribhuvan University, Kathmandu, Nepal. ${ }^{3}$ Institute of Tropical Medicine, Nagasaki University, Nagasaki, Japan. ${ }^{4}$ Epidemiology and Disease Control Division, Department of Health Services, Ministry of Health and Population, Government of Nepal, Kathmandu, Nepal. ${ }^{5}$ Ministry of Health and Population, Government of Nepal, Kathmandu, Nepal. ${ }^{6}$ Institute of Medicine, Tribhuvan University, Kathmandu, Nepal. ${ }^{7}$ Department of Health Services, Ministry of Health and Population, Government of Nepal, Kathmandu, Nepal. ${ }^{8}$ National Public Health Laboratory, Ministry of Health and Population, Government of Nepal, Kathmandu, Nepal. ${ }^{9}$ Walter Reed/AFRIMS Research Unit Nepal (WARUN), Kathmandu, Nepal. ${ }^{10}$ Armed Forces Research Institute of Medical Sciences (AFRIMS), Bangkok, Thailand.

Received: 13 April 2020 Accepted: 2 February 2021

Published online: 18 February 2021

\section{References}

1. Bonell A, Lubell $Y$, Newton PN, Crump JA, Paris DH. Estimating the burden of scrub typhus: a systematic review. PLoS Negl Trop Dis. 2017;11(9): e0005838.

2. Jiang J, Richards AL. Scrub typhus: no longer restricted to the Tsutsugamushi triangle. Trop Med Infect Dis. 2018;3(1):11.

3. Jeromie Wesley Vivian T, Ravi V, Leonard M, Govindakarnavar A, Samir VS, Kamran Z, Tarun B, KSH S, Arun K, Jazeel A, et al. Risk factors for acquiring scrub typhus among children in Deoria and Gorakhpur Districts, Uttar Pradesh, India, 2017. Emerg Infect Dis J. 2018;24(12):2364.

4. Ranjan J, Prakash JAJ. Scrub typhus re-emergence in India: contributing factors and way forward. Med Hypotheses. 2018;115:61-4.

5. Tshokey T, Choden T, Sharma R. Scrub typhus in Bhutan: a synthesis of data from 2009 to 2014. WHO S East Asia J Public Health. 2016;5(2):117-22.

6. Tshokey T, Stenos J, Durrheim DN, Eastwood K, Nguyen C, Vincent G, Graves SR. Rickettsial Infections and Q Fever Amongst Febrile Patients in Bhutan. Trop Med Infect Dis. 2018;3(1):12. https://doi.org/10.3390/tropicalmed301 0012.

7. Brown GW, Shirai A, Gan E, Bernthal P. Antibodies to typhus in eastern Nepal. Trans R Soc Trop Med Hyg. 1981;75(4):586-7.

8. Zimmerman MD, Murdoch DR, Rozmajzl PJ, Basnyat B, Woods CW, Richards AL, Belbase RH, Hammer DA, Anderson TP, Reller LB. Murine typhus and febrile illness, Nepal. Emerg Infect Dis. 2008;14(10):1656-9.

9. Blacksell SD, Sharma NP, Phumratanaprapin W, Jenjaroen K, Peacock SJ, White NJ, Pukrittayakamee S, Day NPJ. Serological and blood culture investigations of Nepalese fever patients. Trans R Soc Trop Med Hyg. 2007; 101(7):686-90

10. EDCD. Guideline on Prevention and Control of Scrub Typhus in Nepal. Kathmandu Nepal: Epidemiology and Disease Control Division (EDCD), Ministry of Health, Nepal; 2016.

11. EDCD. Outbreak reports. Kathmandu, Nepal: Epidemiology and Disease Control Division (EDCD); 2016.

12. Basnyat B. Typhoid versus typhus fever in post-earthquake Nepal. Lancet Glob Health. 2016;4(8):e516-7.

13. Ruang-Areerate $T$, Jeamwattanalert $P$, Rodkvamtook W, Richards AL, Sunyakumthorn P, Gaywee J. Genotype diversity and distribution of Orientia 
tsutsugamushi causing scrub typhus in Thailand. J Clin Microbiol. 2011;49(7): 2584-9.

14. Rodkvamtook W, Ruang-Areerate T, Gaywee J, Richards AL, Jeamwattanalert P, Bodhidatta D, Sangjun N, Prasartvit A, Jatisatienr A, Jatisatienr C. Isolation and characterization of Orientia tsutsugamushi from rodents captured following a scrub typhus outbreak at a military training base, Bothong district, Chonburi province, Central Thailand. Am J Trop Med Hyg. 2011; 84(4):599-607.

15. Masakhwe C, Linsuwanon P, Kimita G, Mutai B, Leepitakrat S, Yalwala S, Abuom D, Auysawasi N, Gilbreath T, Wanja E et al: Identification and Characterization of Orientia chuto in Trombiculid Chigger Mites Collected from Wild Rodents in Kenya. 2018, 56(12).

16. NHRC. Ethical Guidelines for the Care and Use of Animals in Health Research in Nepal. Kathmandu, Nepal: Nepal Health Research Council (NHRC), Government of Nepal; 2005.

17. Paris DH, Shelite TR, Day NP, Walker DH. Unresolved problems related to scrub typhus: a seriously neglected life-threatening disease. Am J Trop Med Hyg. 2013;89(2):301-7.

18. Sharma PK, Ramakrishnan R, Hutin YJF, Barui AK, Manickam P, Kakkar M, Mittal V, Gupte MD. Scrub typhus in Darjeeling, India: opportunities for simple, practical prevention measures. Trans R Soc Trop Med Hyg. 2009; 103(11):1153-8.

19. Mane A, Kamble S, Singh MK, Ratnaparakhi M, Nirmalkar A, Gangakhedkar R. Seroprevalence of spotted fever group and typhus group rickettsiae in individuals with acute febrile illness from Gorakhpur, India. Int J Infect Dis. 2019;79:195-8.

20. Mittal M, Thangaraj JWV, Rose W, Verghese VP, Girish Kumar CP, Mittal M, Sabarinathan R, Bondre V, Gupta N, Murhekar MV. Scrub typhus as a cause of acute encephalitis syndrome, Gorakhpur, Uttar Pradesh, India. Emerg Infect Dis. 2017;23(8):1414-6.

21. George T, Rajan S, Peter J, Hansdak S, Prakash J, lyyadurai R, Mathuram A, Antonisamy B, Ramanathan K, Sudarsanam T. Risk factors for acquiring scrub typhus among the adults. J Global Infect Dis. 2018;10(3):147-51.

22. Thompson CN, Blacksell SD, Paris DH, Arjyal A, Karkey A, Dongol S, Giri A, Dolecek C, Day N, Baker S, et al. Undifferentiated febrile illness in Kathmandu, Nepal. Am J Trop Med Hyg. 2015;92(4):875-8.

23. Adhikari S, Poudel RS, Shrestha S, Lamichhane P. Predictors of mortality in scrub typhus infection requiring intensive care admission in tertiary healthcare Centre of Nepal. Interdiscip Perspect Infect Dis. 2018;2018: 4867958.

24. Candasamy S, Purushothaman J, Paily KP, Pradeep KN, Ayanar E, Athisaya MK, Sundararajan A, Thirumal S, Bhaskar MB. Occurrence of Orientia tsutsugamushi, the etiological agent of scrub typhus in animal hosts and mite vectors in areas reporting human cases of acute encephalitis syndrome in the Gorakhpur region of Uttar Pradesh, India. Vector Borne Zoonotic Dis. 2018;18(10):539-47.

25. Nayak N. Scrub typhus in Nepal. Nepal J Epidemiol. 2016;6(2):563-4.

26. Luce-Fedrow A, Lehman ML, Kelly DJ, Mullins K, Maina AN, Stewart RL, Ge H, John HS, Jiang J, Richards AL. A review of scrub typhus (Orientia tsutsugamushi and related organisms): then, now, and tomorrow. Trop Med Infect Dis. 2018;3(1):8.

27. Michael DL, Abdul Azeez Y, Kriangkrai L, Ahmed R, Kirkvitch C, James WJ. Scrub typhus reemergence in the Maldives. Emerg Infect Dis J. 2003;9(12):1638.

28. Karki S, Shakya P, Cheng AC, Dumre SP, Leder K. Trends of etiology and drug resistance in enteric fever in the last two decades in Nepal: a systematic review and meta-analysis. Clin Infect Dis. 2013;57(10):e167-76.

29. Kim DM, Kim SW, Choi SH, Yun NR. Clinical and laboratory findings associated with severe scrub typhus. BMC Infect Dis. 2010;10:108.

30. Wu Y-C, Qian Q, Magalhaes RJS, Han Z-H, Haque U, Weppelmann TA, Hu WB, Liu Y-X, Sun Y-S, Zhang W-Y, et al. Rapid increase in scrub typhus incidence in mainland China, 2006-2014. Am J Trop Med Hyg. 2016;94(3): 532-6.

31. Varghese GM, Raj D, Francis MR, Sarkar R, Trowbridge P, Muliyil J. Epidemiology \& risk factors of scrub typhus in South India. Indian J Med Res. 2016;144(1):76-81

32. Walker DH. Scrub typhus - scientific neglect, ever-widening impact. N Engl J Med. 2016;375(10):913-5.

33. Dumre SP, Bhandari R, Shakya G, Shrestha SK, Cherif MS, Ghimire $P$, Klungthong C, Yoon IK, Hirayama K, Na-Bangchang K, et al. Dengue virus serotypes 1 and 2 responsible for major denque outbreaks in Nepal: clinical, laboratory, and epidemiological features. Am J Trop Med Hyg. 2017.
34. Dumre SP, Shakya G, Na-Bangchang K, Eursitthichai V, Rudi Grams H, Upreti SR, Ghimire P, Kc K, Nisalak A, Gibbons RV, et al. Dengue virus and Japanese encephalitis virus epidemiological shifts in Nepal: a case of opposing trends. Am J Trop Med and Hyg. 2013;88(4):677-80.

35. KC KP, Shakya G, Malla S, Ghimire P, Paudel B, Dumre SP, Adhikari B: Leptospirosis: one of the neglected infectious diseases in Nepal. In: Proceedings of first international conference on infectious diseases and nanomedicine-2012 (ICIDN-2012): 2013; 2013.

36. Dumre SP, Acharya D, Lal BK, Brady OJ. Dengue virus on the rise in Nepal. Lancet Infect Dis. 2020;20(8):889-90.

37. Dhimal M, Ahrens B, Kuch U. Climate change and spatiotemporal distributions of vector-borne diseases in Nepal--a systematic synthesis of literature. PLoS One. 2015;10(6):e0129869.

38. Pokharel S, Karki M, Acharya B, Marasini B, Arjyal A. Outbreak of acute undifferentiated febrile illness in Kathmandu, Nepal: clinical and epidemiological investigation. BMC Infect Dis. 2020;20(1):89.

39. Tshokey T, John S, Tenzin T, Kinzang D, Bahadur GR, R. GS: serological evidence of rickettsia, Orientia, and Coxiella in domestic animals from Bhutan: preliminary findings. Vector Borne Zoonotic Dis. 2019;19(2):95-101.

\section{Publisher's Note}

Springer Nature remains neutral with regard to jurisdictional claims in published maps and institutional affiliations.
Ready to submit your research? Choose BMC and benefit from:

- fast, convenient online submission

- thorough peer review by experienced researchers in your field

- rapid publication on acceptance

- support for research data, including large and complex data types

- gold Open Access which fosters wider collaboration and increased citations

- maximum visibility for your research: over $100 \mathrm{M}$ website views per year

At BMC, research is always in progress.

Learn more biomedcentral.com/submissions 